\title{
Poorer Physical and Mental Health Status Are Associated with Subsequent Opioid Prescriptions: a U.S. National Study
}

\author{
Anthony Jerant, MD, Alicia Agnoli, MD, MPH, MHS, and Peter Franks, MD \\ Department of Family and Community Medicine, University of California Davis School of Medicine, Sacramento, CA, USA.
}

\begin{abstract}
BACKGROUND: How physical and mental health status relate to receipt of opioid prescription remains unclear, creating uncertainty in minimizing opioid harms while avoiding pain under-treatment.
\end{abstract}

OBJECTIVE: To examine the associations of physical and mental health status with subsequent opioid prescriptions. DESIGN: Observational study of 2005-2015 United States (U.S.) Medical Expenditure Panel Survey data.

PARTICIPANTS: Adult respondents $(N=78,563)$ participating for 2 years. The analyses focused on respondents reporting no opioid prescriptions in year $1(N=65,249)$.

MAIN MEASURES: In the primary analysis, a negative binomial regression yielding adjusted incidence rate ratios (IRRs), the dependent variable was the number of opioid prescriptions in year 2. In two secondary analyses, both logistic regressions yielding adjusted odds ratios (ORs), the dependent variables were receipt of any opioid prescription (versus none) and receipt of $\geq 6$ opioid prescriptions (versus 0-5) in year 2. The key independent variables in all analyses were the SF-12 Physical and Mental Component Summary scores (PCS-12 and MCS12, respectively; higher scores $=$ better health status). All models adjusted for socio-demographics, health-related variables, and year.

KEY RESULTS: Primary analysis. With each 10-point decrement in year 1 PCS-12 or MCS-12 score, there were more opioid prescriptions received in year 2 (adjusted IRRs [95\% CIs] 1.45 [1.39-1.52] and 1.22 [1.16-1.27], respectively). Secondary analyses. With each 10-point decrement in year 1 PCS-12 or MCS-12 score, there were higher odds in year 2 both of receiving any opioid prescription (adjusted ORs 1.23 [1.19-1.28] and 1.11 [1.08-1.15], respectively) and of receiving $\geq 6$ opioid prescriptions (adjusted ORs 1.96 [1.75-2.17] and 1.37 [1.23-1.54], respectively).

CONCLUSIONS: In a nationally representative U.S. sample, both poorer physical and mental health status independently predicted receiving more opioid prescriptions received in a subsequent year, as well as receiving $\geq 6$ prescriptions during the year. Our findings may contribute to a more nuanced picture of the drivers of opioid prescription.

KEY WORDS: analgesics; opioid; health status; pain; surveys and questionnaires; United States.

Received March 12, 2019

Revised July 24, 2019

Accepted September 3, 2019

Published online October 21, 2019
J Gen Intern Med 35(2):554-60

DOI: $10.1007 / \mathrm{s} 11606-019-05401-\mathrm{Z}$

(C) Society of General Internal Medicine 2019

I ncreased clinician prescription of opioid medications in the United States (U.S.) has become a focus of concerntriggering influential appeals for action to reverse the trend. ${ }^{1-4}$ Calls for clinicians to curtail opioid prescription have been driven by concerns regarding discretionary (e.g. excessive or inappropriate) prescription and its potential detrimental impacts on health. ${ }^{5,6}$ Emerging amidst these calls is the need to better identify the drivers of opioid prescriptionto help inform targeted interventions aimed at mitigating the problem. ${ }^{2,3,5}$

Among an array of potential drivers, patient health status might be anticipated to influence subsequent opioid prescriptions. However, the nature of the relationship remains uncertain, since prior studies examining the association of health status with opioid prescription were cross-sectional in nature. ${ }^{7-9}$ The studies also had mixed findings, with some observing poorer health status among prescription in opioid recipients versus non-recipients ${ }^{7,8}$ and others observing better health status among those receiving opioids. ${ }^{9}$ The differences in findings among the studies likely relates in part to the widely varying patient samples examined, all relatively small and highly selected (patients with sickle cell disease, ${ }^{7}$ inflammatory bowel disease, ${ }^{8}$ or assorted chronic painful conditions receiving long-term opioid therapy). ${ }^{9}$

Of potential concern related to the health status-opioid prescription relationship was a U.S. national cross-sectional observational study that found $16 \%$ of individuals with mental health disorders received more than half of all opioids prescribed, adjusting for potential confounders. ${ }^{10}$ While causal mechanisms of this finding could not be examined due to the study design, several explanations that may relate to health status seem plausible and could co-exist. One explanation is that controlling pain may tend to be more difficult in the context of mental health disorders, due to impaired patient coping, heightened pain sensitivity, or other factors, leading clinicians to escalate treatment to opioids. Another potential explanation is that patients with mental health disorders may present their pain (and assess their health status) differently than others, in ways that trigger differential management by 
clinicians. It may also be that at times, clinicians prescribe opioids in part to target the psychological distress (and poorer mental health status) that often co-occurs with physical pain. ${ }^{11,12}$ Others have speculated on such mechanisms to help in explaining the rise in opioid prescription in the U.S. over recent decades, which has paralleled national increases in depression, other mental health diagnoses, and psychological distress and disability. ${ }^{13-21}$

To our knowledge, no prior studies of representative U.S. samples have examined how physical and mental health status at a given time point may influence subsequent receipt of chronic opioid prescriptions. Nationally representative studies exploring this issue could offer guidance to clinicians, patients, and policymakers. Currently, these stakeholder groups have limited evidence to help in balancing fears of inappropriate opioid prescription and opioid-related harms against concerns regarding under-treatment of pain - a quality of care, ethical, and legal dilemma. ${ }^{22-29} \mathrm{We}$ employed data from individuals enrolled for 2 years in the nationally representative 2005-2015 U.S. Medical Expenditure Panel Survey (MEPS) to examine the associations of physical and mental health status measured in participation year 1 with receipt of opioid prescriptions in participation year 2 .

\section{METHODS}

The MEPS is a nationally representative survey of health care use and costs in the U.S. civilian and non-institutionalized population. ${ }^{30}$ The survey is conducted by the Agency for Healthcare Research and Quality (AHRQ) as a subset of the National Health Interview Survey and employs an overlapping panel design. Data are collected for individuals over a 2-year period through baseline and follow-up interviews. The MEPS Household Component collects information including interview language, country of origin, socio-demographic information, health insurance coverage, and health status. Two additional MEPS components, both administered at each follow-up survey and based on respondent self-report, also were examined: the Prescribed Medicines file, which included information on opioid prescription, and the Medical Conditions file, with information reported at each follow-up survey. We utilized MEPS data from these three components for the years 2005-2015. Annual response rates declined from 61.3 to $47.7 \%$ during the study period. ${ }^{31}$

The analytic sample for this study included all participants aged 18 or older who provided baseline data and for whom opioid prescription and health status data were available for their 2-year study participation period. Ethical approval for the study was provided by the University of California Davis Institutional Review Board (exempted status).

\section{Main Measures}

The study measures of physical and mental health status were the 12-item Short-Form (SF-12) Health Survey Physical
Component Summary (PCS-12) and Mental Component Summary (MCS-12) scores, respectively, from MEPS participation year $1 .^{32}$ Both scores range from 0 to 100 , with higher scores indicating better health. Although not a pain measure per se, the PCS-12 includes an item assessing the degree of painrelated functional impairment during the preceding month. Further, generic health status measures including the SF-6D (a preference-based single-index measure which can be derived from SF-12 responses) have been sensitive to changes in opioid use in randomized trials of opioid dependence treatment. $^{33}$

The primary measure of opioid prescription use was the number of opioid prescriptions in participation year 2, a count variable from data in the MEPS Prescribed Medicines file. To further explore the nature of the health status-opioid prescription relationship, we examined the following measures (each again for year 2) in two separate secondary analyses: receipt of any opioid prescription (versus none) and receipt of $\geq 6$ opioid prescriptions (versus $0-5$ ). The $\geq 6$ opioid prescriptions in year 2 category was chosen as a credible proxy for long-term opioid therapy, for which no unequivocal, broadly accepted definition exists. Support for the characterization of $\geq 6$ prescriptions per year as indicating long-term use of opioids comes from the following findings. In the MEPS years we examined, $63 \%$ of those reporting $\geq 6$ prescriptions in year 1 also reported $\geq 6$ prescriptions in year 2 , whereas only $33 \%$ of those reporting $1-5$ prescriptions in year 1 reported any opioid prescriptions in year 2 (7\% reported $\geq 6$ prescriptions). Further, only $7 \%$ of those reporting no opioid prescriptions in year 1 reported $\geq 1$ opioid prescriptions in year 2.

\section{Covariates}

Socio-demographic covariates included age (in years), sex; self-reported race/ethnicity (White, Hispanic, Black, Other); US Census region (Northeast, Midwest, South, West); education level (less than high school, some high school, high school graduate, some college, college graduate); household income level as a percentage of the federal poverty level (FPL) (<100\%, 100-124\%, 125-199\%, 200-399\%, or $\geq 400 \%)$; and health insurance status (uninsured, privately insured, or publicly insured). We further assessed morbidity using a count of eight self-reported chronic conditions (diabetes, hypertension, coronary heart disease, myocardial infarction, cerebrovascular disease, asthma, emphysema, and arthritis). Separately, persons reporting any visit with a diagnosis of any cancer (excluding non-melanoma skin cancer) were coded as having cancer.

\section{Analyses}

We analyzed data using Stata version 15.1 (StataCorp, College Station, TX). In our regression analyses, we utilized longitudinal strata and primary sampling unit identifiers, with survey weights to account for the complex survey design of successive waves of the MEPS. However, reported descriptive 
statistics refer to the study sample and were not adjusted for survey design.

The primary analysis, a negative binomial regression yielding adjusted incidence rate ratios (IRRs) assessed the associations between year 1 physical (PCS-12) or mental (MCS-12) health status (the key independent variables, both continuous) and the number of opioid prescriptions in year 2 (the dependent variable, a count of prescriptions). Negative binominal regression was employed to account for overdispersion of the distribution of opioid prescriptions. ${ }^{34}$ Two separate secondary analyses, both logistic regressions yielding adjusted odds ratios (ORs), assessed the associations between year 1 PCS-12 or mental MCS-12 health status and year 2 receipt of the following: (1) any opioid prescription (versus none) and (2) $\geq 6$ opioid prescriptions (versus $0-5$ ). To explore potential non-linearity of the association between the SF-12 and subsequent opioid prescriptions, the analyses were repeated using the PCS-12 and MCS-12 as categorical variables (both $<40$,

Table 1 Characteristics of the Study Sample in Year 1, by Year 2 Opioid Prescription Category ${ }^{a}$

\begin{tabular}{|c|c|c|c|c|c|}
\hline & $\begin{array}{l}\text { No opioid } \\
\text { prescriptions, } \\
N=\mathbf{5 8 , 7 8 2}\end{array}$ & $\begin{array}{l}1-5 \text { opioid } \\
\text { prescriptions, } \\
N=6083\end{array}$ & $\begin{array}{l}\geq 6 \text { opioid } \\
\text { prescriptions, } \\
N=384\end{array}$ & $p$ value & $\begin{array}{l}\text { Total, } \\
N=65,249\end{array}$ \\
\hline Age, mean (SD) & $47.2(17.7)$ & $47.4(17.3)$ & $54.8(14.7)$ & $<0.001$ & $47.3(17.7)$ \\
\hline Female, no. (\%) & $32,511(55.3)$ & $3780(62.1)$ & $231(60.2)$ & $<0.001$ & $\begin{array}{l}36,522 \\
(56.0)\end{array}$ \\
\hline \multicolumn{6}{|l|}{ Race/ethnicity, no. (\%) } \\
\hline Non-Hispanic White & $29,546(50.3)$ & $3433(56.4)$ & $198(51.6)$ & \multirow[t]{4}{*}{$<0.001$} & \multirow{4}{*}{$\begin{array}{l}33,177 \\
(50.8) \\
14,973 \\
(22.9) \\
11,526 \\
(17.7) \\
5573(8.5)\end{array}$} \\
\hline Hispanic (any race) & $13,772(23.4)$ & 1134 (18.6) & $67(17.4)$ & & \\
\hline Non-Hispanic Black & $10,272(17.5)$ & $1154(19.0)$ & $100(26.0)$ & & \\
\hline Other & $5192(8.8)$ & $362(6.0)$ & $19(4.9)$ & & \\
\hline \multicolumn{6}{|l|}{ Education level, no. (\%) } \\
\hline Less than high school & $5087(8.7)$ & $460(7.6)$ & $48(12.5)$ & \multirow[t]{5}{*}{$<0.001$} & $5595(8.6)$ \\
\hline Some high school & 7296 (12.4) & $750(12.3)$ & $61(15.9)$ & & $8107(12.4)$ \\
\hline High school graduate & $17,289(29.4)$ & $1909(31.4)$ & $129(33.6)$ & & $\begin{array}{l}19,327 \\
(29.6)\end{array}$ \\
\hline Some college & $14,078(23.9)$ & $1640(27.0)$ & $99(25.8)$ & & $\begin{array}{l}15,817 \\
(24.2)\end{array}$ \\
\hline College graduate & $15,032(25.6)$ & $1324(21.8)$ & $47(12.2)$ & & $\begin{array}{l}16,403 \\
(25.1)\end{array}$ \\
\hline \multicolumn{6}{|l|}{ Income as \% FPL, no. (\%) } \\
\hline$<100$ & $9469(16.1)$ & $1082(17.8)$ & $117(30.5)$ & \multirow[t]{5}{*}{$<0.001$} & $\begin{array}{l}10,668 \\
(16.3)\end{array}$ \\
\hline 100 to $<125$ & $3326(5.7)$ & $361(5.9)$ & $32(8.3)$ & & $3719(5.7)$ \\
\hline 125 to $<200$ & $9282(15.8)$ & $914(15.0)$ & $68(17.7)$ & & $\begin{array}{l}10,264 \\
(15.7)\end{array}$ \\
\hline 200 to $<400$ & $17,682(30.1)$ & $1807(29.7)$ & $88(22.9)$ & & $\begin{array}{l}19,577 \\
(30.0)\end{array}$ \\
\hline$\geq 400$ & $19,023(32.4)$ & 1919 (31.5) & $79(20.6)$ & & $\begin{array}{l}21,021 \\
(32.2)\end{array}$ \\
\hline \multicolumn{6}{|l|}{ U.S. region, no. (\%) } \\
\hline Northeast & $9658(16.4)$ & $836(13.7)$ & $46(12.0)$ & \multirow[t]{4}{*}{$<0.001$} & $\begin{array}{l}10,540 \\
(16.2)\end{array}$ \\
\hline Midwest & $12,021(20.5)$ & $1450(23.8)$ & $75(19.5)$ & & $\begin{array}{l}13,546 \\
(20.8)\end{array}$ \\
\hline South & $21,515(36.6)$ & $2343(38.5)$ & $164(42.7)$ & & $\begin{array}{l}24,022 \\
(36.8)\end{array}$ \\
\hline West & $15,588(26.5)$ & $1454(23.9)$ & $99(25.8)$ & & $\begin{array}{l}17,141 \\
(26.3)\end{array}$ \\
\hline \multicolumn{6}{|l|}{ Health insurance, no. (\%) } \\
\hline Any private & $36,713(62.5)$ & $3846(63.2)$ & $163(42.4)$ & \multirow[t]{3}{*}{$<0.001$} & $\begin{array}{l}40,722 \\
(62.4)\end{array}$ \\
\hline Only public & $12,341(21.0)$ & $1480(24.3)$ & $170(44.3)$ & & $\begin{array}{l}13,991 \\
(21.4)\end{array}$ \\
\hline Uninsured & $9728(16.5)$ & 757 (12.4) & $51(13.3)$ & & $\begin{array}{l}10,536 \\
(16.1)\end{array}$ \\
\hline \multicolumn{6}{|l|}{ Health status, mean (SD) } \\
\hline MCS-12 & $\begin{array}{l}49.6(10.1) \\
50.6(9.9)\end{array}$ & $\begin{array}{l}47.2(11.4) \\
49.3(10.8)\end{array}$ & $\begin{array}{l}36.5(12.6) \\
44.5(12.7)\end{array}$ & $\begin{array}{l}<0.001 \\
<0.001\end{array}$ & $\begin{array}{l}49.3(10.3) \\
50.5(10.0)\end{array}$ \\
\hline $\begin{array}{l}\text { Chronic conditions, median } \\
\text { (IQR) }\end{array}$ & $0.93(1.18)$ & $1.17(1.28)$ & $2.04(1.55)$ & $<0.001$ & $0.96(1.20)$ \\
\hline Current cancer, no. (\%) & 1997 (3.4) & $315(5.2)$ & $28(7.3)$ & $<0.001$ & $2340(3.6)$ \\
\hline Opioid prescriptions, mean (SD) & $0.00(0.00)$ & $1.49(0.93)$ & $9.46(7.79)$ & $<0.001$ & 0.19 (1.06) \\
\hline
\end{tabular}

${ }^{a}$ All participants reported no opioid prescriptions in year 1; percentages refer to the sample and are not adjusted for survey characteristics FPL federal poverty level, IQR interquartile range, MCS-12 SF-12 Mental Component Summary, PCS-12 SF-12 Physical Component Summary, SD standard deviation, U.S. United States 
$40 \leq 50,50 \leq 60$, and $\geq 60$ ). The use of the $<40$ and $\geq 60$ categories reflected the relatively small numbers of respondents at the low and high ends of the score ranges.

The models examining the association of the PCS-12 score with subsequent opioid prescriptions adjusted for MCS-12 score; similarly, the models examining the association of the MCS-12 score with opioid prescriptions adjusted for PCS-12 score. All models also included the year 1 values for several other covariates. Socio-demographic characteristics included were age, gender, race/ethnicity category, U.S. Census region, education level, household income level as a percentage of the FPL, and health insurance status. Health-related adjustor variables included the count of eight chronic health conditions, and current cancer diagnosis. All analyses also adjusted for MEPS panel year to account for potential secular temporal trends.

The differences in the number of opioid prescriptions received per one unit change in the PCS-12 or MCS-12 score are small. Thus, we report the differences in the number of opioid prescriptions received per 10-point increment or decrement in PCS-12 or MCS-12 score, corresponding to approximately one standard deviation in each measure. The 10-point increments also exceed the previously reported minimal clinically important differences (MCIDs) for these measures (and for the identically scaled SF-36 measure from which the SF-12 items derive) of 3-6 points. ${ }^{35-37}$

\section{RESULTS}

The analytic sample included 65,249 adult MEPS participants between the years 2005 and 2015 who reported (1) no opioid prescriptions in participation year 1 and who also had (2) no missing physical and mental health status data in year 1 and (3) no missing opioid prescription data in year 2 . The year 1 characteristics of the sample by opioid prescription status in year 2 and overall are shown in Table 1. A total of 58,782 participants $(90 \%)$ reported receiving no opioid prescriptions in year 2, and most of those reporting opioid prescriptions had 1-5 prescriptions. Among the three opioid prescription categories, those who received no opioids had the most favorable physical and mental health status, followed by those who received $1-5$ prescriptions and then those who received $\geq 6$ opioid prescriptions. As compared with those in the other opioid prescription categories, higher proportions of those receiving $\geq 6$ prescriptions were older, female, non-Hispanic Black, less educated, poorer, lived in the South or West, had only public health insurance, and had a current cancer diagnosis (Table 1).

Table 2 shows the findings of the primary and secondary adjusted analyses. In the primary analysis, with 10-point decrements in year 1 PCS-12 or MCS-12, there were more adjusted opioid prescriptions received in year 2 . In the secondary analyses, with 10-point decrements in year 1 PCS-12 or MCS-12, there were higher odds in year 2 both of receiving any opioid prescription and of receiving $\geq 6$ opioid prescriptions (Table 2). As depicted in Figure 1, in both the primary and secondary analyses, the PCS-12 and MCS- 12 were related linearly to the dependent variable.

\section{DISCUSSION}

In a study of a nationally representative sample of adults who received no opioid prescriptions during their first of two MEPS participation years, we found that 10-point decrements in both the year 1 PCS-12 and MCS-12 scores predicted receiving more opioid prescriptions in year 2 . These findings were mirrored in two secondary analyses in which the dependent variables were categorical: receipt of any opioid prescriptions (versus none) and receipt of $\geq 6$ opioid prescriptions (versus 0-5), respectively. In all three analyses, the relationships of the PCS-12 and MCS-12 scores with the dependent variable were essentially linear (Fig. 1). These findings suggest that in the general U.S. population, both poor physical and mental health status are key independent drivers of opioid prescription, with lower scores (i.e., worse physical and mental health status) being associated with receipt of more prescriptions.

Our study was not designed to address the mechanisms of the observed findings. However, the PCS-12 is focused on physical symptoms and functioning and includes one item specifically addressing pain-related interference with home and work activities. Thus, it seems unsurprising that PCS-12 scores would be predictive of subsequent opioid prescriptions, particularly given the emphasis in recent pain management guidelines on targeting functional goals as the key treatment outcome rather than focusing primarily on pain severity (e.g., pain scale scores). ${ }^{5}$

Table 2 Adjusted Associations of Physical and Mental Health Status in Year 1 with Opioid Prescriptions in Year $2(N=65,249)^{\mathrm{a}}$

\begin{tabular}{lll}
\hline \hline $\begin{array}{l}\text { Number of opioid } \\
\text { prescriptions }\end{array}$ & $\begin{array}{c}\text { Adjusted IRR } \\
(95 \% \text { CI })^{\mathrm{b}}\end{array}$ & $p$ value \\
PCS-12 & $1.45(1.39-1.52)$ & $<0.001$ \\
MCS-12 & $1.22(1.16-1.27)$ & $<0.001$ \\
Any opioid prescription & Adjusted OR & $p$ value \\
$\quad$ (versus none) & $(95 \%$ CI) & \\
PCS-12 & $1.23(1.19-1.28)$ & $<0.001$ \\
MCS-12 & $1.11(1.08-1.15)$ & $<0.001$ \\
$\geq 6$ opioid prescriptions & Adjusted OR & $p$ value \\
(versus 0-5) & $(95 \%$ CI) & \\
PCS-12 & $1.96(1.75-2.17)$ & $<0.001$ \\
MCS-12 & $1.37(1.23-1.54)$ & $<0.001$ \\
\hline
\end{tabular}

${ }^{a}$ Analyses adjusted for age, sex, racelethnicity category, education level, income level as a percentage of FPL, U.S. region, health insurance, MEPS panel, a count of chronic health conditions, and presence of cancer, as well as survey characteristics. The PCS-12 analyses also adjusted for MCS-12 score; the MCS-12 analyses also adjusted for PCS-12 score

${ }^{b}$ Per 10-point decrement in PCS-12 or MCS-12 score

CI confidence interval, FPL Federal Poverty Level, IRR incidence rate ratio, OR odds ratio, MCS-12 SF-12 Mental Component Summary, MEPS Medical Expenditures Panel Survey, PCS-12 SF-12 Physical Component Summary, U.S. United States 

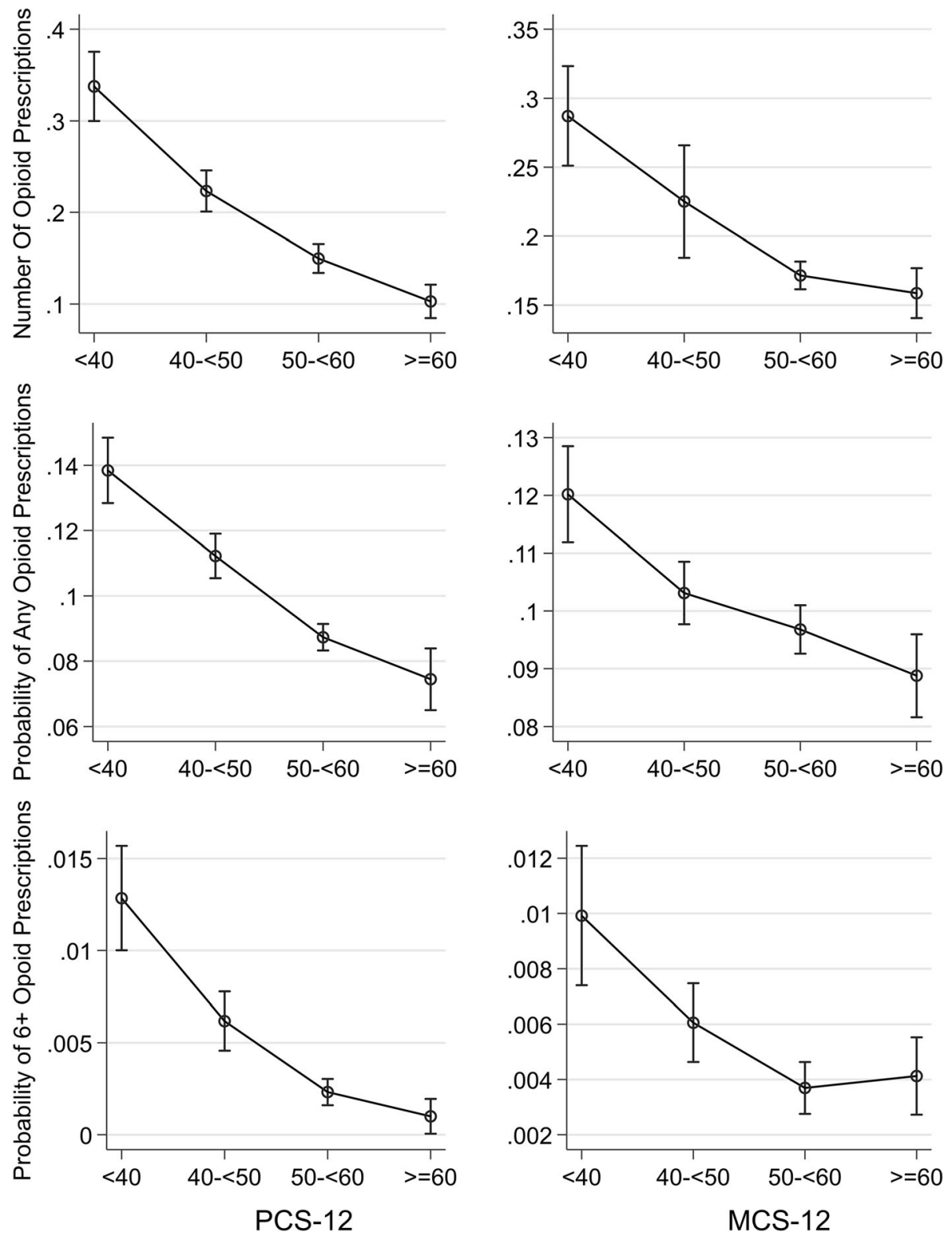

Figure 1 Adjusted associations of year 1 SF-12 Physical Component Summary (PCS-12) and Mental Component Summary (MCS-12) scores (in 10-point increments or decrements) with year 2: number of opioid prescriptions (top row); receipt of any opioid prescription (versus none) (middle row); and receipt of $\geq 6$ opioid prescriptions (versus 0-5) (bottom row). Analyses adjusted for age, sex, race/ethnicity category, education level, and income level as a percentage of federal poverty level, United States region, health insurance, Medical Expenditure Panel Survey panel, a count of chronic health conditions, and presence of cancer, as well as survey characteristics. The PCS-12 analyses also adjusted for MCS-12 score; the MCS-12 analyses also adjusted for PCS-12 score.

Less clear are the reasons for the association of MCS-12 scores with subsequent opioid prescriptions, present despite adjusting for PCS-12 scores. In lieu of a definite explanation, there are several plausible hypotheses as noted previously. Patients with poorer mental health status may have heightened sensitivity to pain or more trouble coping with pain and may also convey and describe pain in more compelling ways than others, including through non-verbal cues. ${ }^{14}$ Additionally, it is possible that clinicians may at times prescribe opioids partly with the goal of alleviating psychological distress, often concurrent with pain. ${ }^{15-21}$ Collectively, these potential explanations for the association of mental health status with subsequent opioid prescriptions represent hypotheses to be examined empirically. Each hypothesis has somewhat different implications for efforts to improve opioid prescription safety and promote deprescribing. For example, if our hypotheses regarding the mechanisms of the association of MCS-12 scores with receipt of opioid prescriptions are confirmed, it might be beneficial to make clinicians aware of the mechanisms and to provide training in safer and more effective approaches to responding to patients' psychological distress. Thus, studies examining the hypotheses in broadly representative samples could provide much-needed guidance to clinicians, patients, and policymakers seeking to reduce discretionary opioid prescription while also avoiding under-treatment of pain. $^{22-29}$ 
Strengths of our study included the use of a nationally representative sample, and the observational cohort (rather than cross-sectional) design. As noted previously, the existing literature has often conflated opioid abuse and misuse with all use of opioids, including as-prescribed. Further, the studies were purely cross-sectional, precluding causal or even temporal inferences, and employed relatively small and nonrepresentative (highly selected) samples. Though we are unable to assess longer-term associations between health status and opioid prescriptions, the examination of relationships over the short (2 years) MEPS participation period was also a strength in that it allowed us to examine a more direct link between the exposure and the outcome, with less opportunity for exogenous confounding than in longer-term studies.

Our study also had some limitations. Non-response to the MEPS may have introduced bias, as the degrees to which receipt of opioid prescriptions or health status may differ among responders and non-responders is unknown. Additionally, opioid prescriptions were self-reported by MEPS participants. To the extent that under- or over-reporting of opioid prescriptions may have occurred, this could bias the findings, particularly if misreporting varied systematically with physical or mental health status, though with uncertain net impact. We did not have information regarding specific mental health diagnoses contributing to the association between MCS-12 scores and subsequent opioid prescriptions. Nonetheless, the MCS-12 measure has been shown to be sensitive in capturing the functional impact of prevalent mental health conditions such as depression. ${ }^{38,39}$ We also lacked information regarding the doses (e.g., morphine-equivalent units) of, indications for, and potential non-medical uses of prescribed opioids. Additionally, we lacked information regarding the timing and sequencing of opioid prescriptions, so it was not possible to discern whether multiple opioid prescriptions in a given MEPS participation year were continuous or separated by off periods. Nonetheless, examining how physical and mental health status in participation year 1 are associated with the number (duration) of opioid prescriptions received in participation year 2, independent of dosage and sequence information, is still worthwhile given studies suggesting more detrimental health effects for longer versus shorter exposure over a given time period. ${ }^{9,40,41}$

In conclusion, in the primary study analysis, we found that 10 -point decrements in physical and mental health status in MEPS participation year 1 were associated with receiving more opioid prescriptions in year 2 , an essentially linear relationship. These findings were closely mirrored in two secondary analyses with categorical dependent variables: receipt of any opioid prescription (versus none) and receipt of $\geq$ 6 opioid prescriptions (versus $0-5$ ) in year 2. That physical health status would predict subsequent opioid prescriptions is unsurprising. The finding for mental health status is less intuitive and suggests the possibility of opioid prescription targeted to psychological distress concurrent with physical pain. Further studies to elucidate the mechanisms underlying the association of mental health status with opioid prescription would be useful, to guide targeted opioid deprescribing efforts that balance avoiding opioid harms with concerns about under-treatment of pain.

Corresponding Author: Anthony Jerant, MD; Department of Family and Community Medicine University of California Davis School of Medicine, Sacramento, CA, USA (e-mail: afjerant@ucdavis.edu).

\section{Compliance with ethical standards:}

Ethical approval for the study was provided by the University of California Davis Institutional Review Board (exempted status).

Conflict of interest: The authors have no conflicts of interest to disclose.

\section{REFERENCES}

1. Sites BD, Beach ML, Davis MA. Increases in the use of prescription opioid analgesics and the lack of improvement in disability metrics among users. Reg Anesth Pain Med. 2014;39:6-12.

2. Alexander LM, Keahey D, Dixon K. Opioid use disorder: a public health emergency. JAAPA. 2018;31:47-52.

3. HHS acting secretary declares public health emergency to address national opioid crisis. October 26, 2017. Available at: https://www.hhs. gov/about/news/2017/10/26/hhs-acting-secretary-declares-publichealth-emergency-address-national-opioid-crisis.html. Accessed August $21,2019$.

4. The opioid crisis in the USA: a public health emergency. Lancet. 2017;390:2016.

5. Dowell D, Haegerich TM, Chou R. CDC Guideline for prescribing opioids for chronic pain-United States, 2016. JAMA. 2016;315:1624-45.

6. Manchikanti L, Kaye AM, Knezevic NN, et al. Responsible, safe, and effective prescription of opioids for chronic non-cancer pain: American Society of Interventional Pain Physicians (ASIPP) Guidelines. Pain Physician. 2017;20:S3-S92.

7. Karafin MS, Singavi A, Hussain J, et al. Predictive factors of daily opioid use and quality of life in adults with sickle cell disease. Hematology. 2018;23:856-63.

8. Anderson A, Click B, Ramos-Rivers C, et al. The association between sustained poor quality of life and future opioid use in inflammatory bowe disease. Inflamm Bowel Dis. 2018;24:1380-8.

9. Dillie KS, Fleming MF, Mundt MP, et al. Quality of life associated with daily opioid therapy in a primary care chronic pain sample. J Am Board Fam Med. 2008;21:108-17.

10. Davis MA, Lin LA, Liu H, Sites BD. Prescription opioid use among adults with mental health disorders in the United States. J Am Board Fam Med. 2017;30:407-17.

11. Torrance N, Lawson KD, Afolabi E, et al. Estimating the burden of disease in chronic pain with and without neuropathic characteristics: does the choice between the EQ-5D and SF-6D matter? Pain. 2014; 155:1996-2004.

12. Bao Y, Sturm R, Croghan Tw. A national study of the effect of chronic pain on the use of health care by depressed persons. Psychiatr Serv. 2003;54:693-7.

13. Linton SJ, Shaw WS. Impact of psychological factors in the experience of pain. Phys Ther. 2011:91:700-11.

14. Turk DC, Okifuji A. What factors affect physicians' decisions to prescribe opioids for chronic noncancer pain patients? Clin J Pain. 1997;13:330-6.

15. Patients with mental disorders get half of all opioid prescriptions. Kaiser Health News. June 26, 2017.

16. Goesling J, Henry MJ, Moser SE, et al. Symptoms of depression are associated with opioid use regardless of pain severity and physical functioning among treatment-seeking patients with chronic pain. J Pain. 2015; 16:844-51

17. Halbert BT, Davis RB, Wee CC. Disproportionate longer-term opioid use among U.S. adults with mood disorders. Pain. 2016;157:2452-7.

18. Sullivan MD, Edlund MJ, Zhang L, et al. Association between menta health disorders, problem drug use, and regular prescription opioid use. Arch Intern Med. 2006;166:2087-93. 
19. Weinberger AH, Gbedemah M, Martinez AM, et al. Trends in depression prevalence in the USA from 2005 to 2015: widening disparities in vulnerable groups. Psychol Med. 2018;48:1308-15.

20. Mojtabai R, Jorm AF. Trends in psychological distress, depressive episodes and mental health treatment-seeking in the United States: 2001-2012. J Affect Disord. 2015;174:556-61.

21. Wittayanukorn S, Gian J, Hansen RA. Prevalence of depressive symptoms and predictors of treatment among U.S. adults from 2005 to 2010. Gen Hosp Psychiatry. 2014;36:330-6.

22. Carvalho AS, Martins Pereira S, Jacomo A, et al. Ethical decision making in pain management: a conceptual framework. J Pain Res. 2018;11:967-76.

23. Greco MT, Roberto A, Corli O, et al. Quality of cancer pain management: an update of a systematic review of undertreatment of patients with cancer. J Clin Oncol. 2014;32:4149-54.

24. Jukic M, Puljak L. Legal and ethical aspects of pain management. Acta Med Acad. 2018;47:18-26.

25. Seers T, Derry S, Seers K, et al. Professionals underestimate patients pain: a comprehensive review. Pain. 2018;159:811-8.

26. Kroenke K, Cheville A. Management of chronic pain in the aftermath of the opioid backlash. JAMA. 2017;317:2365-6.

27. Fishman S, Berger L. The War on Pain. New York, NY: Harper Perennial; 2001.

28. Dineen KK, DuBois JM. Between a rock and a hard place: can physicians prescribe opioid to treat pain adequately while avoiding legal sanction? Am J Law Med. 2016;42:7-52.

29. Serafini M. The physicians' quandary with opioids: pain versus addiction. NEJM Catalyst. Available at: https://catalyst.nejm.org/quandaryopioids-chronic-pain-addiction/. Accessed August 21, 2019.

30. Survey Background - Medical Expenditure Panel Survey. Available at: https://meps.ahrq.gov/mepsweb/about_meps/survey_back.jsp. Accessed August 21, 2019.

31. Response rates - Medical Expenditure Panel Survey (MEPS). Available at: https://meps.ahrq.gov/survey_comp/hc_response_rate.jsp. Accessed August 21, 2019
32. Ware J Jr., Kosinski M, Keller SD. A 12-Item Short-Form Health Survey: construction of scales and preliminary tests of reliability and validity. Med Care. 1996;34:220-33.

33. Nosyk B, Sun H, Guh DP, et al. The quality of eight health status measures were compared for chronic opioid dependence. J Clin Epidemiol. 2010;63:1132-44.

34. Payne EH, Hardin JW, Egede LE, et al. Approaches for dealing with various sources of overdispersion in modeling count data: scale adjustment versus modeling. Stat Methods Med Res. 2017;26:1802-23.

35. Samsa G, Edelman D, Rothman ML, Williams GR, Lipscomb J, Matchar D. Determining clinically important differences in health status measures: a general approach with illustration to the Health Utilities Index Mark II. Pharmacoeconomics. 1999;15:141-55

36. Diaz-Arribas MJ, Fernandez-Serrano M, Royuela A, et al. Minimal clinically important difference in quality of life for patients with low back pain. Spine. 2017;42:1908-16.

37. Jayadevappa R, Cook R, Chhatre S. Minimal important difference to infer changes in health-related quality of life - a systematic review. J Clin Epidemiol. 2017;89:188-98.

38. Salyers MP, Bosworth HB, Swanson JW, Lamb-Pagone J, Osher FC. Reliability and validity of the SF-12 health survey among people with severe mental illness. Med Care. 2000;38:1141-50.

39. Vilagut G, Forero CG, Pinto-Meza A, et al. The mental component of the short-form 12 health survey (SF-12) as a measure of depressive disorders in the general population: results with three alternative scoring methods. Value Health. 2013;16:564-73

40. Mojtabai R. National trends in long-term use of prescription opioids. Pharmacoepidemiol Drug Saf. 2018;27:526-34

41. Brat GA, Agniel D, Beam A, et al. Postsurgical prescriptions for opioid naive patients and association with overdose and misuse: retrospective cohort study. BMJ. 2018;360:j5790.

Publisher's Note: $\quad$ Springer Nature remains neutral with regard to jurisdictional claims in published maps and institutional affiliations. 